\title{
Trombosis venosa profunda de miembros inferiores asociada a una triple deficiencia enzimática: antitrombina III, proteína $\mathrm{C}$ y proteína S. Reporte de caso
}

\section{Deep venous thrombosis of lower members associated with a triple enzyme deficiency: antithrombin III, protein C and protein S. Case} report

Franco-Díaz de León, Raúl; Gamboa-Solís, Raúl; Sánchez-Díaz de León, José Antonio; Laurel-Razo, Alado

Raúl Franco-Díaz de León raul.franco@edu.uaa.mx Universidad Autónoma de Aguascalientes, México Raúl Gamboa-Solís doc_gamboa@hotmail.com Instituto Mexicano del Seguro Social, México José Antonio Sánchez-Díaz de León jatba98@mail.com Universidad Autónoma de Aguascalientes, México Alado Laurel-Razo aldolaurel06@gmail.com Universidad Autónoma de Aguascalientes, México

\footnotetext{
Lux Médica

Universidad Autónoma de Aguascalientes, México

ISSN: 2007-1655

Periodicidad: Cuatrimestral

vol. 16, núm. 48, 2021

luxmedica.editorial@gmail.com

Recepción: 12 Noviembre 2020

Aprobación: 07 Mayo 2021

URL:

https://revistas.uaa.mx/index.php/luxmedica/article/view/3335

Autor de correspondencia: raul.franco@edu.uaa.mx

Poítica de acceso abierto La Revista Lux Médica proporciona un acceso abierto a su contenido, basado en el principio de que ofrecer un acceso libre a las investigaciones ayuda a incrementar el intercambio global del conocimiento. La LM no cobra ni cobrará ningún cargo a sus lectores por concepto de suscripción, ni a los autores por enviar, procesar o publicar sus artículos. Como condición de publicación, los autores acuerdan liberar sus derechos de autor bajo una licencia compartida, específicamente la licencia de Creative Commons Reconocimiento-NoComercial-Compartir Igual 4.0 Internacional Esta licencia permite a cualquier persona compartir, copiar y redistribuir el material en cualquier medio o formato bajo los siguientes términos: - Dar crédito al autor del texto - No hace
}

Resumen: La Enfermedad Tromboembólica Venosa comprende dos trastornos, el Tromboembolismo Pulmonar y la Trombosis Venosa Profunda (TVP). Esta última se asocia, entre otras causas, a deficiencias enzimáticas del organismo, las cuales favorecen el desarrollo de la enfermedad y sus complicaciones, siendo el síndrome postrombótico la más frecuente. Presentamos el caso de un paciente masculino de 34 años que acude a consulta por presentar aumento de volumen, edema, tensión muscular, dolor y claudicación de ambas extremidades pélvicas, a quien se le diagnosticó Trombosis Venosa Profunda Fémoro-Ilíaca Bilateral asociada a una triple deficiencia enzimática de Antitrombina III, Proteína C y Proteína S de la coagulación.

Palabras clave: Trombosis Venosa Profunda, Antitrombina III, Proteína C y $\mathrm{S}$.

Abstract: Venous Thromboembolic disease includes two disorders, pulmonary thromboembolism and deep vein thrombosis (DVT). The latter is associated, among other causes, with enzymatic deficiencies of the organism, which favor the development of the disease and its complications, with Postthrombotic Syndrome being the most common. We present a case report from a 34-years-old male patient who came for examination due to an increased volume, edema, muscle tension, pain, and claudication of both pelvic limbs being diagnosed previously of bilateral femoro-iliac deep vein thrombosis associated with a triple enzymatic deficiency of antithrombin III, Protein C and Protein S from coagulation.

Keywords: Deep Vein Thrombosis, Antithrombin III, Protein C and $S$. 
uso del material con propósitos comerciales - No transformar o modificar el material. Los autores ceden el derecho de la primera publicación a esta revista, pero conservarn sus derechos de autor, de tal forma que pueden realizar otros acuerdos contractuales independientes y adicionales para la distribución no exclusiva de la versión del artículo publicado en esta revista ( por ejemplo, incluirlo en un repositorio institucional o publicarlo en un libro) siempre que indiquen claramente que el trabajo se publicó por primera vez en ésta.

\section{(1) (2)}

Esta obra está bajo una Licencia Creative Commons Atribución-

NoComercial-CompartirIgual 4.0 Internacional.

Cómo citar este artículo: Franco-Díaz de León , R., Gamboa-Solís,

R., Sánchez-Díaz de León, A., Laurel-Razo, A. Trombosis venosa

profunda de miembros inferiores asociada a una triple deficiencia enzimática: antitrombina III, proteína $\mathrm{C}$ y proteína $\mathrm{S}$. Reporte de caso. Lux Médica. Recuperado a partir de https://revistas.uaa.mx/ind ex.php/luxmedica/article/view/3323

\section{INTRODUCCIÓN}

La Trombosis Venosa Profunda (TVP) es un tipo de Tromboembolismo Venoso con predominio en extremidades inferiores. Se trata de un padecimiento en el que se forma un trombo dentro de una vena del sistema venoso profundo que puede asociarse a múltiples factores de riesgo, los cuales se pueden agrupar en la triada de Virchow conformada por estasis venosa, hipercoagulabilidad y lesión o daño vascular. ${ }^{1}$ Dichos factores de riesgo deben ser considerados al momento de realizar la historia clínica y la exploración del paciente. Entre ellos se incluyen las trombofilias hereditarias o genéticas, las cuales representan las causas más frecuentes de Tromboembolismo Venoso en pacientes menores de 50 años. Estas se clasifican en: mutaciones de pérdidas funcionales (deficiencias enzimáticas de Antitrombina III, Proteína $S$ y Proteína C de la coagulación son algunas de las más importantes) y mutaciones de ganancias funcionales (como la mutación de protrombina G20210A y factor V Leiden). ${ }^{1,2}$

Es necesario considerar cada uno de los factores mencionados al momento de realizar el diagnóstico y al ofrecer el tratamiento, ya que esto permite promover la prevención de recurrencia y desarrollo de Síndrome Postrombótico(SPT), el cual representa la complicación más común a largo plazo $(>50 \%)$ y se caracteriza por la presencia de dolor, edema, prurito, aumento de volumen, hiperpigmentación cutánea (dermatitis ocre) y la formación de úlcera venosa en los casos más severos de SPT (presente en 5-10\% de los pacientes). ${ }^{3,4}$

La deficiencia de Antitrombina III es una trombofilia poco prevalente (0.02-0.2\%). Se trata de un anticoagulante endógeno que inhibe la serpina, la cual a su vez inhibe la trombina, factor de coagulación Xa, IXa, XIa y XIIa. Su déficit se asocia a un riesgo 16 veces mayor de desarrollar trombosis venosa profunda en un primer episodio y 1.6 a 3.7 veces su recurrencia. Se ha encontrado una relación del $80 \%$ entre los pacientes con valores bajos de antitrombina III y la mutación del gen SERPINC1. Al igual que en la antitrombina III, la deficiencia de Proteína C y Proteína $S$ son trombofilias con una baja prevalencia $(0.14-0.5 \%)$. $5,67,8$

La proteína $\mathrm{C}$ de la coagulación actúa inhibiendo los cofactores solubles $\mathrm{V}$ y VIII de la coagulación. Se ha encontrado una relación entre la deficiencia de esta proteína y la mutación del gen PROC del cromosoma 2q14.3. Por su parte, 
la Proteína $S$ es una glucoproteína dependiente de la vitamina $\mathrm{K}$ que funciona como cofactor de la proteína $\mathrm{C}$ una vez que se ha activado. Ambas deficiencias se adquieren de manera autosómica dominante. ${ }^{7,9}$

\section{PRESENTACIÓN DEL CASO}

Se trata de un paciente masculino de 34 años, originario del estado de Aguascalientes, México. Acude al servicio de urgencias en el año 2013 por presentar aumento de volumen y edema de miembros inferiores. Durante la exploración física en el servicio de angiología de la institución se encuentra tensión muscular en ambas extremidades inferiores y dolor de moderada intensidad en piernas y muslos, el cual alcanza la región inguinal por lo que se decide ingresar a la unidad de cuidados intensivos.

De la historia clínica: no presenta antecedentes heredofamiliares de importancia, reside en un municipio del mismo estado, de ocupación fontanero, tabaquismo y alcoholismo lo refiere negativo, no adicto a drogas, no convive con animales domésticos, realiza ocasionalmente ejercicio, no refiere antecedentes patológicos de alguna enfermedad previa, ningún antecedente quirúrgico, no ingesta de medicamentos, no alergias y niega traumatismos.

Su padecimiento actual lo inicia dos días antes de su ingreso con dolor en ambas piernas y muslos, aumento de volumen, endurecimiento de extremidades y claudicación bilateral. Sus signos vitales se encuentran dentro de parámetros normales, presenta buena coloración tegumentaria, bien hidratado, faringe sin compromiso, cuello simétrico, sin masas palpables, tráquea central, pulsos carotídeos presentes, no soplos, ruidos cardiacos de buena intensidad y sin arritmias, campos pulmonares bien ventilados, buena amplexión y amplexación, sin datos de condensación pulmonar, abdomen blando sin dolor, no se palpan masas, peristalsis presente y normal, sin soplos arteriales, extremidades superiores con pulsos presentes, sin edema ni aumento de volumen, extremidades inferiores con aumento de volumen, edema en regiones inguinales, sin tumoraciones $\mathrm{ni}$ adenomegalias, edema en ambos muslos y piernas, tensión muscular, pulsos femorales, poplíteos y tibiales bilaterales presentes, signos de Olow Prat y Homans presentes, buen llenado capilar en dedos de ambos pies, movilidad normal y reflejos normales. A la exploración con USG Doppler se encuentra flujo venoso disminuido a nivel femoral.

Se ingresa al paciente en el Servicio de Angiología del hospital con diagnóstico de Trombosis Venosa Profunda Fémoro-Iliaca Bilateral, iniciando protocolo para descartar causas de deficiencias enzimáticas. El paciente ingresa sin complicaciones de flegmasia alba ni cerúlea dolens y sin presentar datos de tromboembolia pulmonar. Se solicitan estudios de laboratorio básico con: biometría hemática completa, química sanguínea, tiempos de coagulación y enzimas de Antitrombina III, Proteína C y Proteína $S$ de la coagulación (Tabla $1)$. 
Tabla 1.

Resultados del examen de laboratorio

\begin{tabular}{|c|c|c|}
\hline Reactivos & $\left|\begin{array}{l}\text { Valores } \\
\text { del } \\
\text { Paciente } \\
(m g / a L)\end{array}\right|$ & $\begin{array}{l}\text { Valores Normales } \\
(m g / d L)\end{array}$ \\
\hline Antitrombina III & 42.3 & \begin{tabular}{|l|l|} 
Mujeres & $243-380$ \\
Hombres & $260-378$ \\
\end{tabular} \\
\hline Proteina C de la Coagulación & 0.42 & \begin{tabular}{|l|l|} 
Mujeres & $1.67-3.16$ \\
Hombres & $1.76-2.90$ \\
\end{tabular} \\
\hline \multirow{2}{*}{ Proteina S de la Coagulación } & \multirow{2}{*}{1.37} & \begin{tabular}{|l|l|}
\multirow{2}{*}{ Mujeres } & $9.46-$ \\
& 21.3 \\
\end{tabular} \\
\hline & & \begin{tabular}{|l|l|} 
Hombres & $\begin{array}{l}13.5- \\
24.1\end{array}$ \\
\end{tabular} \\
\hline Anticuerpos Anti-cardiolipinas IgG & $\begin{array}{l}3.3 \\
\text { GPL } \\
\mathrm{ml}\end{array}$ & $\begin{array}{l}\text { Normales o } \\
\text { Negativos 0-15 } \\
\text { GPL / ml }\end{array}$ \\
\hline Anticuerpos Anti-cardiolipinas IgM & 5.9 & $\begin{array}{l}\text { Normales o } \\
\text { Negativos 0-15 } \\
\text { MPL/ml }\end{array}$ \\
\hline \multicolumn{3}{|c|}{$\begin{array}{l}\text { Resultado de laboratorio obtenido en el año } 2013 \text {. Se observa } \\
\text { una deficiencia triple enzimática: Antitrombina III, Proteina C y } \\
\text { Proteína S de la coagulación. Los Anticuerpos Anticardiolipinas } \\
\text { IgG e IgM son negativos. }\end{array}$} \\
\hline
\end{tabular}

El paciente recibió tratamiento los primeros ocho días en internamiento intrahospitalario con reposo total. Se le administró heparina de alto peso molecular convencional por vía intravenosa a dosis de $1000 \mathrm{UI} /$ hora por infusión continua. Al sexto día de internamiento se inició con la administración por vía oral de anticoagulante acenocumarina a dosis de impregnación (sexto día - 12 $\mathrm{mg}$; séptimo día $-8 \mathrm{mg}$; octavo día $-4 \mathrm{mg}$ ), dándose de alta a su domicilio al noveno día con indicación de mantener una buena hidratación, vendaje elástico de miembros inferiores, medidas de higiene venosa estricta y la ingesta de acenocumarina a dosis de 1-4 mg vía oral cada 24 horas, con el control de Tiempo de Protrombina (TP), el cual deberá de reportase mediante estudio de laboratorio entre 24 y 30 segundos, con un INR de 2-2.5 y un porcentaje de actividad de 40-60\%. Hasta la actualidad, durante siete años, mantiene estas indicaciones con ingesta de acenocumarina para llevar un control de anticoagulación con los parámetros presentados anteriormente.

Se le realiza al paciente en junio de 2020 un estudio de USG Doppler Vascular Periférico Tipo Venoso Bilateral de miembros pélvicos inferiores desde pliegue inguinal hasta maléolo medial en posición de pie en escala de grises, Doppler a color y Doppler pulsado con ayuda de maniobras de inspiración profunda y valsalva, así como dorsiflexión de ortejos.

Los resultados que arrojó el estudio se muestran a continuación: Extremidad Inferior Izquierda: En la vena iliaca externa, la vena femoral común, vena femoral profunda, vena femoral en sus diferentes segmentos (Figura 1) y en la Vena poplítea (Figura 2) se muestran defectos de llenado hipoecoico relacionado a trombosis residual mostrando colapso parcial tras la maniobra de compresión. Se identifica duplicación de la vena femoral (Figura 2) que abarca todos sus segmentos mostrando ambas con material hipoecoico en su interior relacionado 
a trombosis residual. Tras la aplicación Doppler Color existe llenado parcial de lumen vascular con evidencia de reflujo multisegmentario tras la maniobra de valsalva. Otro hallazgo del miembro pélvico izquierdo corresponde a la incompetencia de dos venas perforantes en pierna medial en territorio Sherman y Cockett III (Figura 3). Se encuentra también unión safeno-poplítea presente con material hipoecoico en su interior relacionado a trombosis residual, vena perforante incompetente en tercio medio de pierna posterior en territorio May y edema de los tejidos blandos desde pliegue de rodilla asociado a celulitis que es más evidente en tercio medio y distal de pierna (Figura 4).

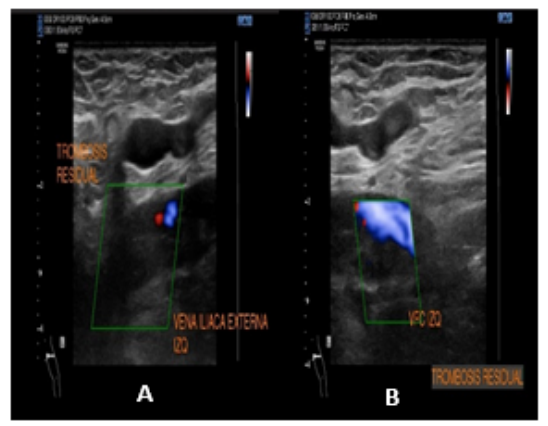

Fig. 1. A. Vena Iliaca Externa izquierda con defecto de llenado hipoecoico asociado a trombosis residual. B. Vena Femoral Común izquierda con defecto de llenado hipoecoico asociado a trombosis residual.

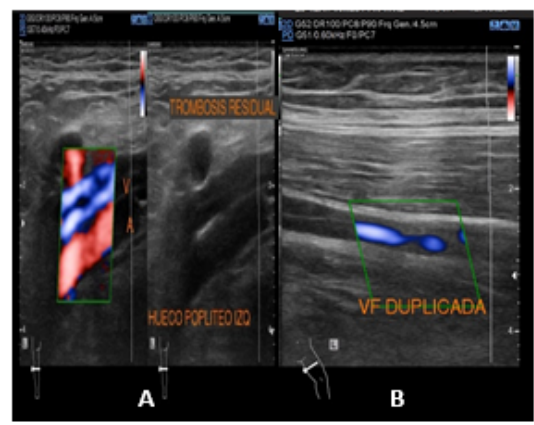

Fig. 2 A. Vena Poplitea izquierda con defecto de llenado hipoecoico asociado a trombosis residual. B. Duplicación de Vena Femoral izquierda con material hipoecoico asociado a trombosis residual.

Figuras 1 y 2.

Fig. 1. A. Vena Iliaca Externa izquierda con defecto de llenado hipoecoico asociado a trombosis residual. B. Vena Femoral Común izquierda con defecto de llenado hipoecoico asociado a trombosis residual.

Fig. 2 A. Vena Poplítea izquierda con defecto de llenado hipoecoico asociado a trombosis residual. B. Duplicación de Vena Femoral izquierda con material hipoecoico asociado a trombosis residual. en ambas.

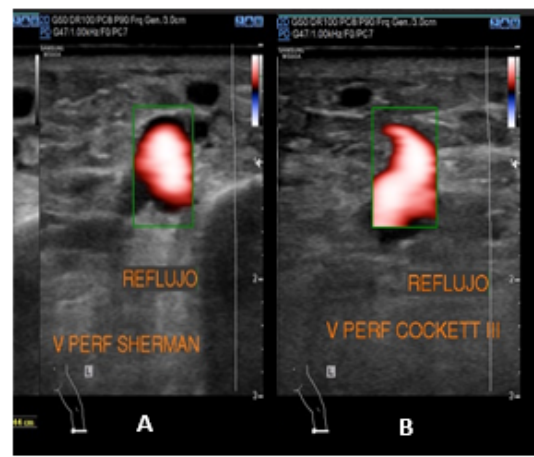

Fig. 3. A. Incompetencia de vena perforante (cara medial de pierna izquierda en territorio Sherman). B. Incompetencia de vena perforante (cara medial de la pierna izquierda en territorio Cockett III).

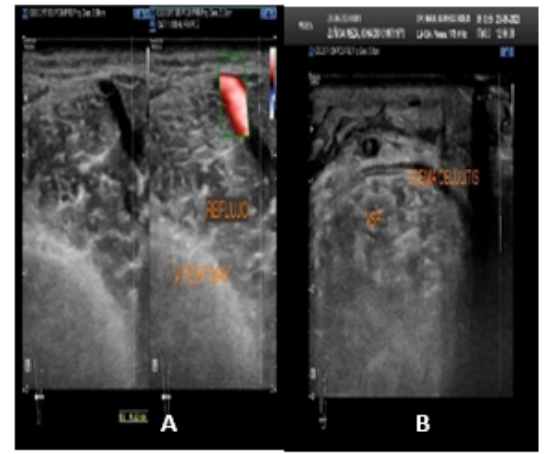

Fig. 4. A. Vena perforante con incompetencia en tercio medio de pierna izquierda posterior en territorio May. B. Edema de tejidos blandos asociado a celulitis en tercio medio y distal de piema izquierda.

Figuras 3 y 4 .

Fig. 3. A. Incompetencia de vena perforante (cara medial de pierna izquierda en territorio Sherman).

B. Incompetencia de vena perforante (cara medial de la pierna izquierda en territorio Cockett III).

Fig. 4. A. Vena perforante con incompetencia en tercio medio de pierna izquierda posterior en territorio May. B. Edema de tejidos blandos asociado a celulitis en tercio medio y distal de pierna izquierda.

Extremidad Inferior Derecha: se encuentra la vena iliaca externa, la vena femoral común, la vena femoral profunda, vena femoral en sus diferentes segmentos con defectos de llenado hipoecoicos relacionado a trombosis residual mostrando colapso parcial tras la maniobra de compresión (Figura 5). Se identifica un contenido de material hipoecoico en la vena poplítea derecha (distal 
en canal de Hunter) y una duplicación de la vena femoral en todo su recorrido también con contenido hipoecoico, ambas relacionadas a trombosis residual (Figura 6).

La unión venosa safeno-poplítea se encuentra presente con material hipoecoico en su interior relacionado a trombosis residual con la presencia de reflujo venoso en la valoración de Doppler Color. Además, se aprecia extensión craneal de la vena safena parva hasta el muslo posterior en su tercio distal (Figura 7). Se encuentra la vena safena parva en recorrido habitual sobre su ojo safeno sobre la línea media de la pierna posterior con calibre normal, solo mostrando engrosamiento concéntrico en tercio medio y proximal (Figura 8). Las venas tibiales anteriores y posteriores sin alteraciones. Es posible observar una vena perforante incompetente en tercio medio de pierna posterior en territorio May (Figura 9). Existe edema de los tejidos blandos desde pliegue de rodilla asociado a celulitis que es más evidente en tercio medio y distal de pierna (Figura 10).

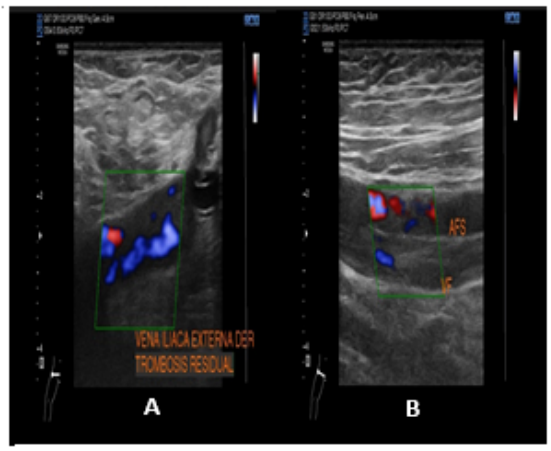

Fig. 5. A. Vena Iliaca Externa derecha con defecto de 1 lenado asociado a trombosis residual. B. Vena Femoral derecha con defecto de llenado hipoecoico asociado a trombosis residual.

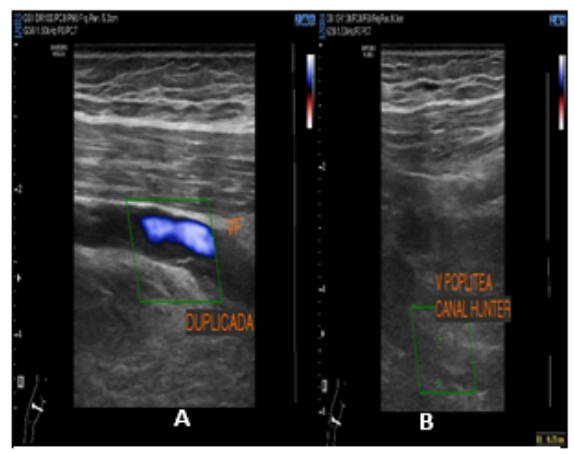

Fig. 6. A. Vena Femoral derecha con defecto de llenado asociado a trombosis residual. B. Vena Poplitea derecha distal (canal de Hunter) con defecto de llenado hipoecoico asociado a trombosis residual.

\section{Figuras 5 y 6}

Fig. 5. A. Vena Iliaca Externa derecha con defecto de llenado asociado a trombosis residual.

B. Vena Femoral derecha con defecto de llenado hipoecoico asociado a trombosis residual.

Fig. 6. A. Vena Femoral derecha con defecto de llenado asociado a trombosis residual. B. Vena

Poplítea derecha distal (canal de Hunter) con defecto de llenado hipoecoico asociado a trombosis residual.

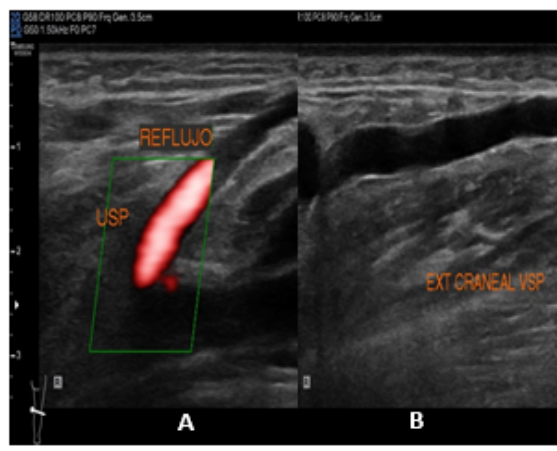

Fig. 7. A. Unión Venosa safeno-poplítea con defecto de llenado asociado a trombosis residual y presencia de reflujo venoso. B. Extensión craneal de la vena safena parva hasta el muslo posterior en su tercio distal.

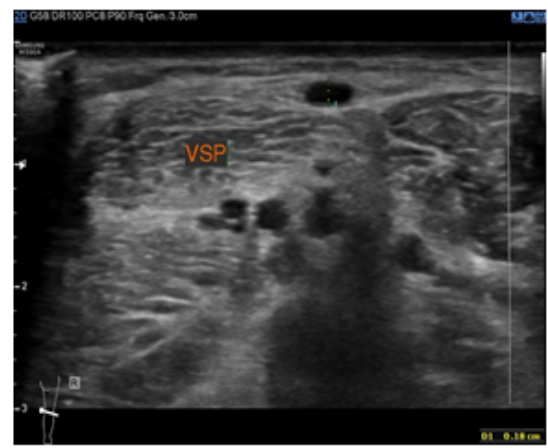

Fig. 8. Engrosamiento concéntrico en tercio medio y proximal de la vena safena parva.

Figuras 7 y 8 .

Fig. 7. A. Unión Venosa safeno-poplítea con defecto de llenado asociado a trombosis residual y presencia de reflujo venoso. B. Extensión craneal de la vena safena parva hasta el muslo posterior en su tercio distal. Fig. 8. Engrosamiento concéntrico en tercio medio y proximal de la vena safena parva. 


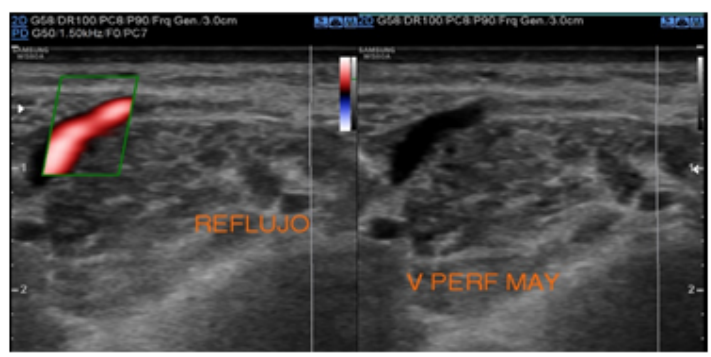

Fig. 9. Vena perforante incompetente en tercio medio de pierna posterior (territorio May) con presencia de reflujo venoso.

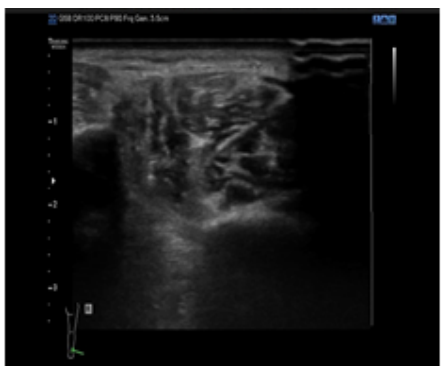

Fig. 10. Edema de tejidos blandos asociado a celulitis en tercio distal de pierna derecha.

Figuras 9 y 10.

Fig. 9. Vena perforante incompetente en tercio medio de pierna posterior (territorio May) con presencia de reflujo venoso.

Fig. 10. Edema de tejidos blandos asociado a celulitis en tercio distal de pierna derecha.

Con lo anterior se obtiene la impresión diagnostica de Trombosis Venosa Residual Bilateral en Sistemas Profundos, Incompetencia Venosa Combinada (superficial-profunda-perforante) de la Extremidad Inferior Izquierda y Derecha, y Edema Bilateral de tejidos blandos desde pliegues de rodilla con celulitis, siendo esto más evidente en tercio medio y distal de ambas piernas. No se aprecian paquetes varicosos.

\section{DISCUSIÓN}

La enfermedad tromboembólica venosa constituye una de las principales causas de morbi-mortalidad, afectando aproximadamente a 1 de cada 1,000 personas al año y se asocia con múltiples factores de riesgo en pacientes quirúrgicos y no quirúrgicos. Este trastorno clínico cursa con dos manifestaciones clínicas: Tromboembolia Pulmonar (3-4 personas por cada 10,000) y Trombosis Venosa Profunda. ${ }^{10}$ En este artículo se presenta el caso de un paciente masculino de 34 años de edad originario y residente del estado de Aguascalientes, México, quien acude a consulta por presentar síntomas y signos aparentes de una TVP, como lo son el edema, aumento de volumen, dolor moderado, claudicación bilateral y tensión muscular en ambas extremidades inferiores, desde la zona inguinal hasta los tobillos. Sin embargo, durante la historia clínica no presenta factores de riesgo aparentes para presentar un cuadro de Trombosis Venosa Profunda a nivel Femoroiliaca Bilateral, como lo son aquellas circunstancias que desencadenan la interacción de los tres eventos que componen la Triada de Virchow: 1) estasis venosa (hospitalizaciones previas, viajes prolongados), 2) estado de hipercoagulabilidad (antecedentes de TVP, ingesta de medicamentos, vejez) y 3) lesión vascular (cirugías previas, traumatismos, uso de marcapasos o uso de catéter venoso central); así como un estado de deshidratación, antecedentes de sepsis, mordedura de insectos y animales venenosos, vasculitis o enfermedades autoinmunes. ${ }^{1}$ Debido a esto, se sospechó de un estado de hipercoagulabilidad primaria por la presencia de una trombofilia hereditaria o congénita (principal causa de TVP en pacientes menores de 50 años). ${ }^{2}$

Para solicitar estudios de laboratorio, siempre es necesario realizar con anterioridad una completa historia clínica, una anamnesis adecuada y descartar cualquier causa de trombofilia adquirida. En este paciente, no había antecedentes de TVP, ni factores de riesgo que orientaran a una etiología no hereditaria, por 
lo que se debe descartar que su manifestación se deba a factores genéticos, por lo que se optó por solicitar estudios de laboratorio, donde se recomienda que los resultados se realicen en dos ocasiones en pacientes que no tengan factores de riesgo para establecer el diagnóstico definitivo. A este paciente se le solicitó un estudio laboratorial para determinar los niveles de Antitrombina III, Proteína C y Proteína $S$ de la coagulación, los cuales fueron reportados como bajos. Asimismo, se descartó que la causa sea debido a un Síndrome Antifosfolipídico (SAF) que es una enfermedad autoinmune caracterizada por la presencia de Anticuerpos Antifosfolípidos aumentando el riesgo de formación de coágulos y cuyas manifestaciones son principalmente Trombosis Venosa Recurrente, teniendo una prevalencia estimada en 1-5\% de la población, siendo más frecuente en pacientes en edad avanzada y en mujeres embarazadas. Para confirmar el diagnóstico de SAF es necesario por lo menos una manifestación clínica y que el laboratorio demuestre la persistencia de Anticuerpos Antifosfolípidos: Anticoagulante lúpico, Anticardiolipina IgG o IgM y o Anti-b2GPI IgG o IgM) durante al menos 12 semanas, en este caso, los resultados fueron negativos para la presencia de Anticuerpos Anticardiolipinas IgG e IgM. ${ }^{11}$

Con base en los resultados obtenidos, se llegó a la impresión diagnóstica de Trombosis Venosa Profunda Fémoro-Iliaca Bilateral debido a una Trombofilia Congénita o Hereditaria por triple deficiencia enzimática de Antitrombina III, Proteína C y $S$ de la coagulación, que lo llevó a un estado de hipercoagulabilidad primaria y una posterior TVP, donde no presenta complicaciones como Tromboembolia Pulmonar (TEP), Flegmasia Alba Dolens (Síndrome Compartimental) o Flegmasia Cerúlea Dolens (Gangrena Venosa). Debido a que una de las complicaciones más frecuentes de la TVP es el Síndrome Postrombótico (hasta en un $50 \%$ de los pacientes), el tratamiento bien abordado debe ser prioridad para evitar que se manifieste, y en caso de que se presente, evitar la complicación más severa del mismo, que ocurre en un 5-10\% de los pacientes. ${ }^{4}$ Al paciente se le indicó un tratamiento farmacológico inmediato (administración de heparina de alto peso molecular convencional IV a dosis de $1000 \mathrm{UI} /$ hora por infusión continua). A partir del sexto día de internamiento se inició con la administración por vía oral de acenocumarina a dosis de impregnación (sexto día - $12 \mathrm{mg}$; séptimo día - $8 \mathrm{mg}$; octavo día $-4 \mathrm{mg}$ ) y al darse de alta a su domicilio (a partir del noveno día, posterior a su estabilización) se le indicó la ingesta de acenocumarina a dosis de $1-4 \mathrm{mg}$ vía oral cada 24 horas, con el control de Tiempo de Protrombina (24y 30 segundos, con un INR de 2-2.5 y un porcentaje de actividad de 40-60\%.). Aunado a su tratamiento farmacológico, también se le indicaron medidas de higiene venosa estricta (ejercicio), una adecuada hidratación y el uso vendaje elástico de miembros inferiores. Lo que sí presenta en la actualidad es un síndrome postrombótico demostrado por Ultrasonografía Doppler, la cual se considera como el método diagnóstico de elección para la TVP y el Síndrome Postrombótico. ${ }^{12}$ El Síndrome Postrombótico (SPT) es una manifestación que se presenta en más del $50 \%$ de los pacientes con TVP y se caracteriza por una serie de signos y síntomas como edema, dolor en las extremidades y, en el peor de los casos, la presencia de ulceras varicosas. Es causada por una insuficiencia venosa crónica e hipertensión venosa posterior a un evento trombótico venoso. No es exclusivo de las extremidades inferiores, sin embargo, sí es la zona más frecuente de esta patología. Parte del tratamiento 
de la TVP es evitar que se llegue a presentar este síndrome o que, si no puede evitarse, sus manifestaciones no sean incapacitantes para el paciente. Estudios han demostrado que la presencia de una TVP proximal o Iliofemoral es un factor de riesgo para desarrollar SPT, teniendo una incidencia máxima a los dos años posteriores al evento trombótico. Una forma de prevenir este síndrome es la extracción directa del coágulo a través de un catéter en la vena afectada (trombectomía mecánica) o mediante la administración de fármacos infundidos por una vena del pie o directamente en el sitio del coágulo mediante catéter y control de rayos $\mathrm{X}$ (trombólisis fármaco-mecánica). ${ }^{10} \mathrm{El}$ diagnóstico de SPT suele ser mediante la clínica, ya que los pacientes acuden a consulta médica por presentar alguna sintomatología como dolor, edema, lesiones dérmicas, etc. Este diagnóstico puede apoyarse mediante Ultrasonografía Doppler y Doppler Color para observar si existe alguna alteración en el sistema venoso, como reflujo. ${ }^{13}$ Existen diversas escalas que se han investigado en diversos estudios que permiten diagnosticar y clasificar la severidad del SPT, entre ellas están: Clasificación de Widmer, Clasificación Clínico-Etiológico-Anatómico-Patológico (CEAP), Puntuación de Gravedad Clínica Venosa (VCSS), Escala de Brandjes, Medida de Ginsberg, Escala de Villalta y la Escala de Villalta informada por el paciente. Hasta la fecha, aún no se ha estipulado un "Gold Standard" ni una escala para la clasificación del SPT. Aún existe una variación considerable en diversos estudios que no han permitido que se llegue a un método diagnóstico general, sin embargo, y entre las diferentes escalas diagnósticas, la que ha tenido mejores resultados es la Escala de Villalta. No obstante, aún no se determina su especificidad ni sensibilidad, por lo que la terapéutica empleada para un paciente con SPT es aún un reto médico. ${ }^{14}$

\section{CONCLUSIÓN}

La Trombosis Venosa Profunda representa la principal causa de embolia pulmonar, además de estar relacionada con diversas complicaciones que alteran el bienestar y el estilo de vida del paciente, siendo el síndrome postrombótico la más frecuente de ellas. Su diagnóstico debe realizarse considerando la etiología multicausal de esta entidad descrita en la triada de Virchow, la cual permite estudiar las diversas causas que pueden provocar esta alteración. A pesar de ser poco frecuentes, las deficiencias enzimáticas corresponden a un estado de hipercoagulabilidad y deben ser sospechadas en el paciente al momento de realizar su historia clínica siempre que se descarten otras causas o factores de riesgo relacionados a estasis venosa, lesión endotelial o un estado de hipercoagulabilidad en la sangre. Un tratamiento y diagnóstico oportuno favorecen el estado del paciente y pueden ser la diferencia entre un pronóstico favorable y uno desfavorable.

\section{REFERENCIAS}

1. Miao J, Naik G, Muddana S, Li X, Bhimasani S, Mitchell R, et al. An uncommon case of lower limb deep vein thrombosis with multiple etiological causes. Am J Case Rep. 2017; 18:313 - 316. 
2. Hotoleanu C. Genetic risk factors in venous thromboembolism. Adv Exp Med Biol. 2016; $906: 253-272$.

3. Kahn SR. The post-thrombotic syndrome. Hematology Am Soc Hematol Educ Program. 2016; 2016 (1) : 413 - 418.

4. Pikovsky O, Rabinovich A. Prevention and treatment of the post-thrombotic syndrome. Thromb Res. 2017; 164:116 - 124.

5. Sokol J, Timp JF, Le Cessie S, Van Hylckama-Vlieg A, Rosendaal F, Kubisz P, Cannegieter S, Lijfering W. Mild antithrombin deficiency and risk of recurrent venous thromboembolism: results from the MEGA follow-up study. J Thromb Haemost. 2018; 16:680-688.

6. Bauer K, Nguyen-Cao T, Spears JB. Issues in the Diagnosis and Management of Hereditary Antithrombin Deficiency: A Review. Annals of Pharmacotherapy. 2016; doi: $10.1177 / 1060028016651276$

7. Vargas A. Trombofilias hereditarias: el perfil de pruebas necesarias. Revista de hematología. 2019; 20(2):79-85.

8. Bravo C, Vicente V, Corral J. Management of antithrombin deficiency: an update for clinicians. Expert Rev Hematol. 2019; 12 (6) : 397 - 405.

9. Dinarvand P, Moser K. Protein C Deficiency. Arch Pathol Lab Med. 2019; $143(10): 1281-1285$.

10. Robertson L, McBride O, Burdess A. Pharmacomechanical thrombectomy for iliofemoral deep vein thrombosis. Cochrane Database of Systematic Reviews. 2016; doi:10.1002/14651858.CD011536.pub2.

11. Linnemann B, Hart C. Laboratory diagnostics in thrombophilia. Hamostaseologie. 2019; $39(1): 49-61$.

12. Al-Thani $\mathrm{H}$, El-Menyar A, Asim M, Kiliyanni AS. Clinical presentation, management, and outcomes of deep vein thrombosis based on doppler ultrasonography examination. Angiology. 2015; 67 (6) :587 - 595.

13. Busuttil A, Lim CS, Davies AH. Post thrombotic syndrome. Adv Exp Med Biol. 2016; $906: 363-375$.

14. Wik HS, Enden TR, Ghanima W, Engeseth M, Kahn SR, Sandset PM. Diagnostic scales for the post-thrombotic syndrome. Thromb Res. 2018; 164:110-115. 